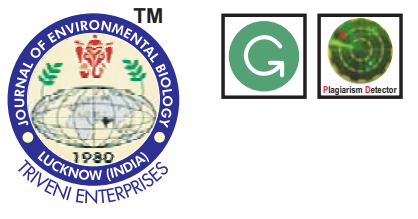

\title{
Isolation of plasmid from a Gram negative bacteria Aeromonas dhakensis strain F2S2-1 and its partial characterization
}

\author{
Authors Info \\ M. Nadiga, ${ }^{1,2}$ V.V. Vaidyanathan \\ and T. Thayumanavan ${ }^{2 *}$ \\ 'Syngene International Ltd \\ Bangalore, India \\ 'Department of Biotechnology, \\ Dr. G. R Damodaran College of \\ Science, Civil Aerodrome Post, \\ Coimbatore - 641014, India
}

*Corresponding Author Email : thayumanavan@yahoo.com

Key words

Aeromonas dhakensis

DNA nicking enzyme

Endonuclease

Plasmid sequencing

Publication Info

Paper received: $\quad 30.06 .2016$

Revised received: $\quad 12.08 .2016$

Re-revised received: 20.01 .2017

Accepted:

28.01.2017

\begin{abstract}
Aim: A plasmid was isolated from Aeromonas dhakensis strain F2S2-1 to understand their function in the host. The main objective of the study was to isolate and sequence the plasmid, predict genes and proteins encoded and to experimentally prove their biochemical function.

Methodology: The Aeromonas dhakensis strain F2S2-1 plasmid was sequenced by Next Generation Sequencing (NGS) on Illumina Miseq platform. Bioinformatics analysis was carried out to predict the putative gene and encoded protein functions. The NspV like endonuclease was recombinantly expressed in Escherichia coli, partially purified and characterized for activity on plasmid substrates.
\end{abstract}

Results: Plasmid sequencing and bioinformatics analysis predicted the presence of NspV like endonuclease.Recombinant expression, purification and characterization of NspV like endonuclease revealed the Nicking endonuclease activity of the protein with plasmid substrates.

Interpretation: The NspV like endonuclease described in this study has DNA nicking activity. this study would help in understanding better survival of Aeromonas $\mathrm{sp}$. in its ecosystem and the role of host plamids.

Identification and purification of an independently replicating plasmid from Aeromonas dhakensis strain F2S2-1

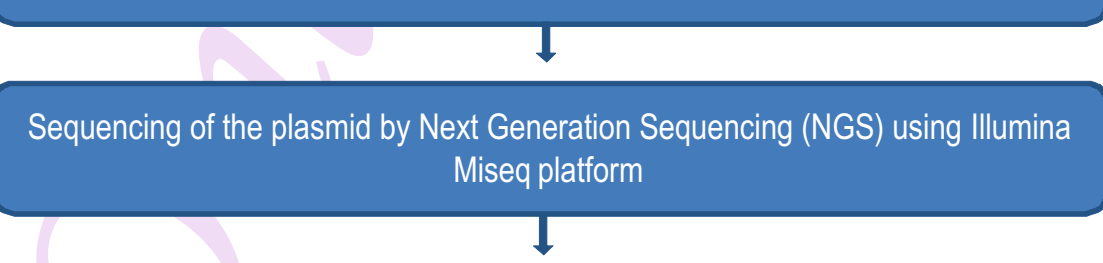

Bioinformatics identification of two separate Restriction - Modification Systems on the plasmid, namely, Cfr9l like and NspV like RM systems

NspV like endonuclease was cloned, expressed recombinantly in Escherichia coli, purified and partially characterized for activity on plasmid substrates

Restriction pattern of the NspV like endonuclease on plasmid substrates points to it being a Nicking Endonuclease 


\section{Introduction}

Aeromonas species are Gram negative, predominantly motile bacteria distributed in aquatic ecosystems across the world. They are also found in varied physical and biological eco systems like aquatic, fish, food, domesticated pets, birds, ticks, insects and to lesser extent natural soils. The species is dominated by Aeromonas hydrophila, Aeromonas salmonicida and to a lesser extent by Aeromonas caviae, Aeromonas sobria, Aeromonas tecta, Aeromonas aquorium, Aeromonas bivalvium, Aeromonas sharmana, Aeromonas simiae etc (Janda and Abbott, 2010). Aeromonas sp. cause fin rot and tail rot in fish, red leg disease in frogs and wound infection in animals. In humans, Aeromonas sp. cause gastroenteritis in children and immunecompromised patients and wound / soft tissue infections (Janda and Abbott, 1996). Hence it is important to study this organism to understand its biology.

Nearly, 3500 Restriction-modification systems are reported in literature till date. These are classified into Type I, Type II, Type III, Type IV systems depending on the protein structure and function (Roberts et al., 2003). Advantage of R-M systems to the bacterial / viral host is less clear but they are described as selfish genetic elements (Kobayashi, 2001). Their presence is known to decrease the viral replication efficiencies in the bacterial host (Pigoud and Jeltsch, 1997; Luria and Human, 1952) and function as defense for bacterial host against bacteriophage infections. Pre-dominant class of Type II restriction enzymes recognize and cut within a typically a 6-bp recognition site producing either blunt ends or sticky, overhanging ends. Type II restriction enzymes are widely used in Biotechnology industry for cloning and DNA modification / engineering.

Nicking endonucleases (NEases) are similar to restriction endonucleases but cleave only one strand of the DNA, where as Type II restriction endonucleases cleave both strands (Higgins et al., 2001). NEases invariably recognize nonpalindromes for their mode of action. NEases can be frequent cutters or rare cutters like homing endonucleases (HEases) (Chan et al., 2011). NEases have wide applications like isothermal DNA amplification, cloning of DNA, studying DNA repair etc. (Zheleznaya et al., 2009). Reported here is a study on the isolation and sequencing of a plasmid PAH-F2S2-1 from Aeromonas dhakensis strain F2S2-1, the cloning - expression and partial purification of $\mathrm{NspV}$ like endonuclease and characterization of its Nickase like function.

\section{Materials and Methods}

Isolation of strain and plasmids : Aeromonas dhakensis strain F2S2-1 was isolated from skin surface of Indian oil sardine (Sardinella longiceps) by Aeromonas selection media (Himedia). The strain was observed to host a plasmid during agarose gel analysis and was purified by plasmid mini prep kit (Qiagen). All the plasmids used in the present study were purified using plasmid mini prep kit(Qiagen)
Sequencing of the plasmid pAH-F2S2-1 and prediction of ORFs : The plasmid was sequenced by Next Generation Sequencing (NGS) on Miseq platform (Illumina) with 2 X 250 bp paired end reads. A total of 233424 reads were obtained, which were assembled by CLC Genomics workbench (Ver. 8.5). The final sequencing to fill the gaps in DNA sequence of the plasmid was carried out by Sanger sequencing on ABI3730XL (ABI). The sequenced plasmid was finally determined to be $10483 \mathrm{bp}$ in length. The ORF prediction was carried out by VNTI (Ver.11), as well as PlasMapper (Ver. 2.0) (Xiaoli et al., 2004).

Cloning of NspV like RM genes : The NspV like methylase was cloned first into Ndel and Kpnl sites of pACYC-Duet vector using the primers NspV-like-M-Ndel-FP - 5' TAA GAA GGA GAT ATA CATATGAGTAAA GAGAAAATT GAG 3' and NspV-like-M-KpnlRP 5' TTT ACC AGA CTC GAG GGT ACC TCA CTA TTA TGC CGT CCA ATC AAT AGA ATT C $3^{\prime}$. After cloning methylase, the NspV like endonuclease was cloned into BamHI and Notl sites of the same vector with the primers NspV-like-E-BamHI-FP 5' CAT CAT CAC AGC CAG GAT CCC ATG GTT TTA ACT CTG ATG 3'and NspV-like-E-Notl-RP - 5' GTT CGA CTT AAG CAT TAT GCG GCC GCC TAT CAT TAT CAT TATAAAAAG GCA GCC CGA TA 3'. All the cloning work was carried out in $E$. coli host DH5alpha.

Expression and purification of NspV like endonuclease : The plasmid pACYC-Duet1 with NspV like methylase and endonuclease were transformed into E. coli BL21(DE3) electrocompetent cells and the protein was induced with IPTG (Sigma). The induced protein was purified with batch processing by Ni-NTA resin (GE health care) affinity purification according to manufacturer's instruction. The purified protein was dialyzed against $50 \mathrm{mM}$ Tris- $\mathrm{HCl}, \mathrm{pH} 8.0$ to remove the Imidazole and stored in $-80^{\circ}$ for assays.

Protein staining and western blotting : The affinity purified protein was resolved on $4-12 \%$ Bis-Tris gel (Thermofisher) with $1 \mathrm{X}$ MES running buffer. The protein was stained with simply blue stain (Thermofisher). For western blot, the protein was transferred to nitrocellulose membrane (PALL life sciences) and developed with 1:1000 penta His mouse primary antibody (Qiagen) and 1:2000 goat anti-mouse secondary antibody (Santa Cruz Biotechnologies). The blot was developed with ECL chemi luminescence kit (GE health care) with Bio rad imaging system.

Endonuclease assays : Approximately, 1ug of affinity purified endonuclease was incubated with $2 \mathrm{ug}$ of test DNAin $1 \mathrm{X}$ cut smart buffer (NEB- $50 \mathrm{mM}$ potassium acetate, $20 \mathrm{mM}$ tris-acetate, $10 \mathrm{mM}$ magnesium acetate, $100 \mathrm{ug}^{-1} \mathrm{ml} \mathrm{BSA}, \mathrm{pH} 7.9$ ) for 2 hrs at 37 degree and the restriction profile was checked on $1 \%$ TAE agarose gel. The lambda DNA and phi x 174 circular DNA used in the assay were procured from Clontech, USA.

\section{Results and Discussion}

The sequencing of plasmid pAHF2S2-1 resulted in a final, circular plasmid of 10483 bp (Genbank Accession KX863708 and KX863709). The annotation of the plasmid 


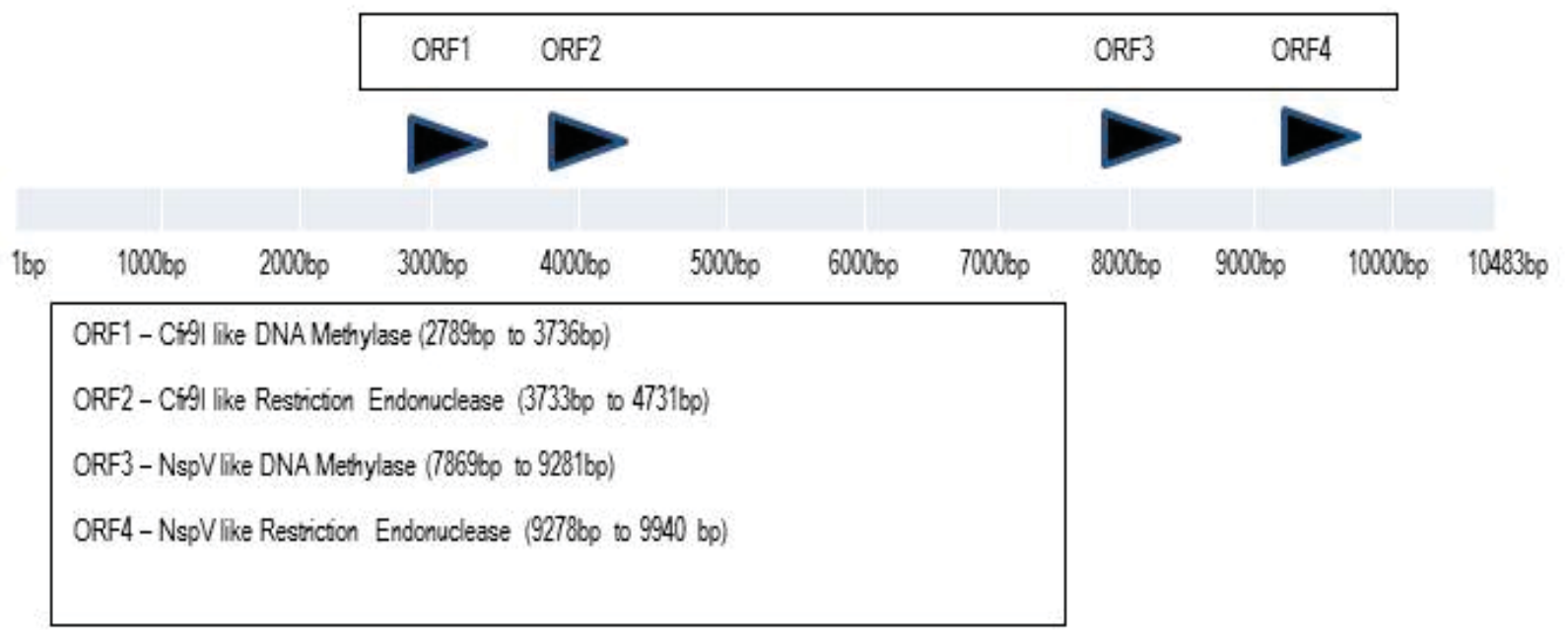

Fig.1 : Layout of the plasmid isolated from Aeromonas dhakensis strain F2S2-1 and the ORF annolation

A. Nucleoode sequence of NspVlike DNA Mehylase

ATGCCGTCCAATCAATAGAATTCAATATGTGGTTIAATCGGTCTTTTTCATCCCAGAAAATCATGGTGATATAGCTCCCAAAACCGGTTGGATTGAAGCTTATCAAGCGCATCCAAAGCATCCTGCTC AAGATCAAAAGAGAGGAAATATACCGTATCATCAAAAACAACAGGTTGTITCAATGGTCAACAAGGGTAAAGGATAGCITITATATAACCCGCAAATAGCTATTICCATGGTTGAATGTATATCAC CGACCCCAAAAACTGAAAACCTAGGGTCTITTATATATAACGCTCTTICTCCATCAAGCTTGTCAGCATGAGATATTAAATAGCGCCACGCTTIAGGGTATTTAGTTICAATGTATGATGTATCTTCACC AACACGTTTGGGTGACTATAACAAATCTATTATCCCACCCTTTCCCACTACCCACTCGAGAGCCTTICAATAAAGGGTAAACTACCTCAACCTCAATATCTACAACATCACCATAACCGTAACCAGCACA CCATCATGTGTTTAGCTCCATAACAGATGATGCGTCGTGCTTCATTCCTGACCTCCATTITITGGGGGGGCTGAAACAAATCGACTTGATAACTCAAATGTATCTATATCACTTACAAGAAACCCTTTCC TATGACCACACAACCGCCCAGTCTTATCACTGAAAGATTCAAAAACTTATAGTCATACGATGGCTTAACCTGTGAATCTATTCGCATATAAAAAAGCAAGCATCCACAGATGCGCCAAACTCTTTCTIGC ATCAACGGTATTATCTCTGAGTAATAACATCCTATTTATGCTTCTCAACATAAGAAAGTATTTCCTGGCCACTGCTGTCTTAAGCATGATTGCTATATCAATTTTTTTCCTGGCGACCATTGGAGCATATC AATCATCATCCACTCTGATATGTCAAAATTTGCCTACCAGTATCGCGTCCAACCCGCCAAGACCAACCACATTGTCTITTCTGGAACGTIATAACTATCAATTTACTCTGGACGGTGTGGTCACCCAT GGCAAGTTCCCCAAAACAAGCAGATTACCATCCACATCAGACGACTTACCTGCCCAGTCATAACGAAAAAAGTCACCATGCTCGATAGATACTACATTTGGCGAGGGTGACCACGCAACGCCCCCTCAA GCACATCGAGATAATCACTGTTAATGTCAATACCATAAACTTTCTCGTGGATGAGAAGTAATTCACTGATGCAATAACAAATGCCCCAACACCACAAGTGGCTCAATAATAACATCAGGAGAAACCCCTA AAGTAACCAAACGCTCACAGGCTATCTCTGCTAATTCTAGCGGCGTTGGAAATCACCAAACTCAATTTTTCTTACTCATAA

\section{B. Amino acid sequence of NspVlike DNA Mehylase}

MSKEKIEFGDFQTPLELAEIACERLVTLGVSPDVIETTCGVGAFVASVNYFSSTKKVYIDINSDYLDVEGALRGHPRQNWSIEHGDFFRYOWAGKSSDVDGNLLLGNLPWVTNTVQSKDSYNVEKONWG LGGLDAITGKANFDISEWMMIDMKWWSPGKKIDAIMLKTAVARKILSWEKHINGCYYSEITVDARKEFGASVDACFFIMRIDSQVIPSYDYKVFESFSDKTGRLCGHRKGFLVSDIDTFELSSRFVSAPPKWWRSGM KHDASSVMEEKQHDGVLVNGYGDWDIEVEWYPLKGGSRVGSGKGWDNRFVIVTQERVGEDTSYIETKYPKAWRYLISHADKLDGRKSVIYKKNPRFSVFGVGEYTFKPWIAICGLYKKRSFTLVEPIDNKPWF DDTVFLSFDLEQDALDALDKLQSKPVLGAISSMIFWDEKRPIKATILNSIDWTA

C. Nucleodide sequence of NspVlike endonudease ATGGTTTAACTCTGATGAATTATGTCGTGAGGCGCGGGACTTTCTCGTCAGGAATCTGTTCATAATGAACCGACATATTGGTGTIACGGATGGAAAGGCAGTGGAACATATTAGAGCAGAAGTT AGAAATACCTTTAGCGAGGTATCAGTIGAGGTGGTAATTCAGCAAGAGGGATGACTTCCTAGTTAAATGTGACATGAAAGTIACAAGTGCGAGGCAGCCTCAGTCTTCATGCCCGTCAGATCT GCCAGACAAAAGGTGTATGGGTGGGGTATGGTCTTATAATTTTGTCTATGATAAATCAGATCCCCTGAAAATAGAACGGCGAGATTGAATATATCTAGCACCATATATGTCGAACOGAATAAAACAGCT GACTACCAGCTGACATCTAGTATGCTCAGGTACTGATAATAATGGTTCAGAAGCAGATCTTATGCGTATTIATGGATAAAAACCTTCCTGTGATGATATAGGTGCTAGGGATTAGCAAGGGAAATAT TGAGAGATAGGCCTCAAATAGGCTGCCTTACAATATCAAATGCTTGCAATGGCGACTTCAGTATACAAGGGCTATGGAGTGGGTGGTAATTATGATGGTGTTCACGCTATTIATCGGGCTGCCTTITAT AATGATAG

D. Amino acid sequence of NspVlike endonuclease

MVLTMNLCREARDFSRQESVHNEPTLFVIDGKAVGTYLEQKFRNYLLARYQFEVGNSARGIDFPSLNVDMKVTSARQPQSSCPFRSARQKVYGLGYGIIFYYKSOSPENRTARLNISSTIYVEPNKTADYQL TSSIAQVLDNNGSEADLIALFMDKN_PVDDIGARDLAREILRDRPQIGQTISNALQWRLQYTRAIEWAGNDGVHAIYRAAFL.

Fig.2 : Nucleotide and amino acid sequence of putative NspV like methylase and endonuclease from Aeromonas dhakensis strain F2S2-1 plasmid 
A

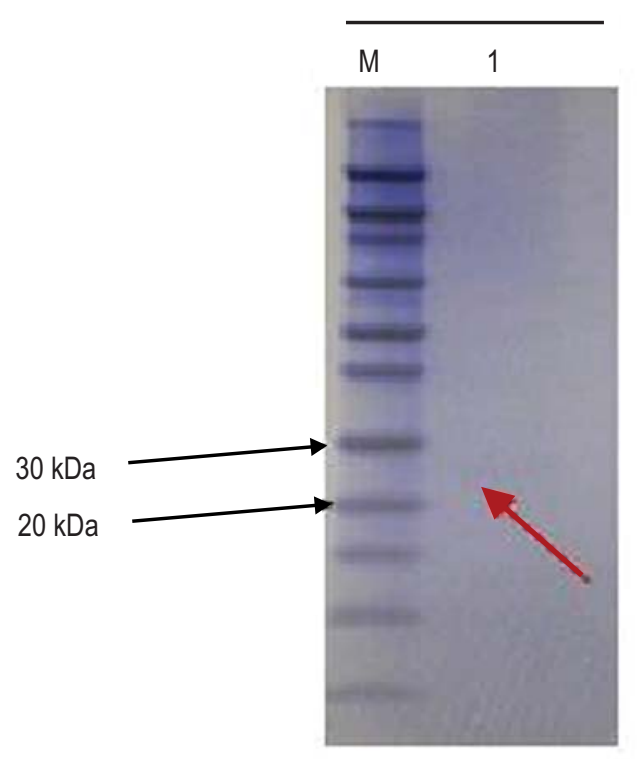

B

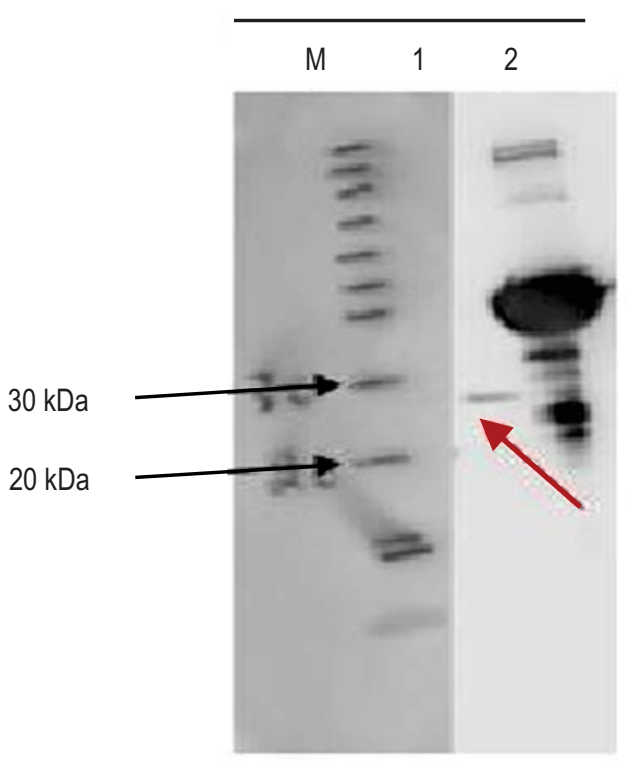

Fig. 3 : Western blot of purifed NspV like endonucleas showing as expression in Ecoil lysare (lane M-) Themofsher Novex Protein marker (35, 10, 15, 20, 30, 40, 50, 60, 80, 110, 160, 260, kDa) .

Lane 1 - Restriction endonuclease expressed (predicted protein MW - $24845 \mathrm{kda}$, shown by red arrow)

Lane 2 - His tag positive control protein A. Protein staining B. Anti His western blot

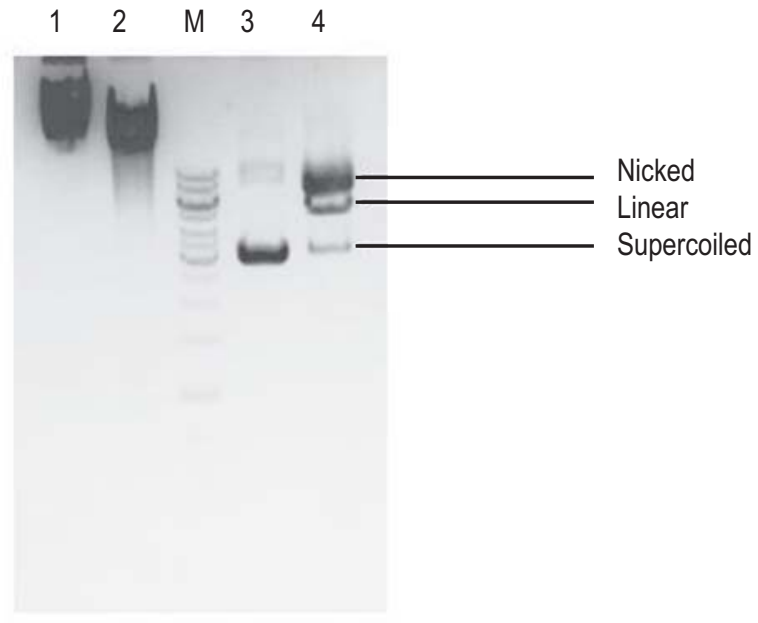

Fig.4 : Restriction profile of lamda DNA and phi x 174 DNA cut with NspV like endonuclease.

Lane 1 - Uncut lamda DNA Lane 2- Lamda DNA cut with NspV like endonuclease Lane M-Fermentas DNA ladder $(0.25,0.5,0.75,1,1.5$, $2,2.5,3,3.5,4,6,8,10 \mathrm{~kb})$

Lane 3- Uncut phi x 174 DNA Lane 4-phi x 174 DNA cut with NspV like endonuclease

revealed the presence of two Restriction-Modification systems similar to Cfr9l and NspV type II restriction enzymes. The Cfr9l like DNA methylase was 1048 bp long and the corresponding endonuclease was 999bp. NspV like DNA methylase was 1413bp long and its endonuclease was 663 bp long. The structure of RM systems are given in Fig.1. and the nucleotide and amino acid sequence of NspV like R-M system is shown in Fig. 2.

NspV like endonuclease expressed and purified from E. coli was confirmed for protein expression by protein staining and anti his Western Blot is shown in Fig. 3.

Sequencing and annotation of the plasmid pAH-F2S21 from Aeromonas dhakensis revealed the presence of two R-M systems. The two R-M systems were Cfr9l like and NspV like respectively. NspV like DNA methylase revealed the presence of conserved motif PDXnEPT (where, $n=1$ to 30 residues) present in many Type II restriction enzymes with the consensus motif being PDXn(D/E)XK (Pigoud and Jeltsch, 1997). The NspV like DNA endonuclease revealed the presence of conserved motif DXnEQK present in some of the Type I restriction endonucleases (Murray, 2000; Davies et al., 1999). The secondary structure prediction with Jpred 4 (Drozdetskiy et al., 2015) revealed that it was structurally close to NspV enzyme.

DNA cleavage of $\mathrm{NspV}$ like endonuclease from Aeromonas dhakensis revealed that it digests lambda DNA and Phi X 174 DNA. The cleavage of lambda DNA was less efficient than Phi X 174 (Fig. 4.). The NspV like endonuclease cleaves the Phi X 174 DNA, pACYC DUET and pTZ57R resulting in predominantly a nicked form (Fig. 4, 5, 6) with formation of a small quantity of linear form. The NspV like restriction endonuclease the present study failed to digest a fragment containing the NspV recognition site TTCGAA(unpublished results). 
$\begin{array}{lllll}M & 1 & 2 & 3 & 4\end{array}$

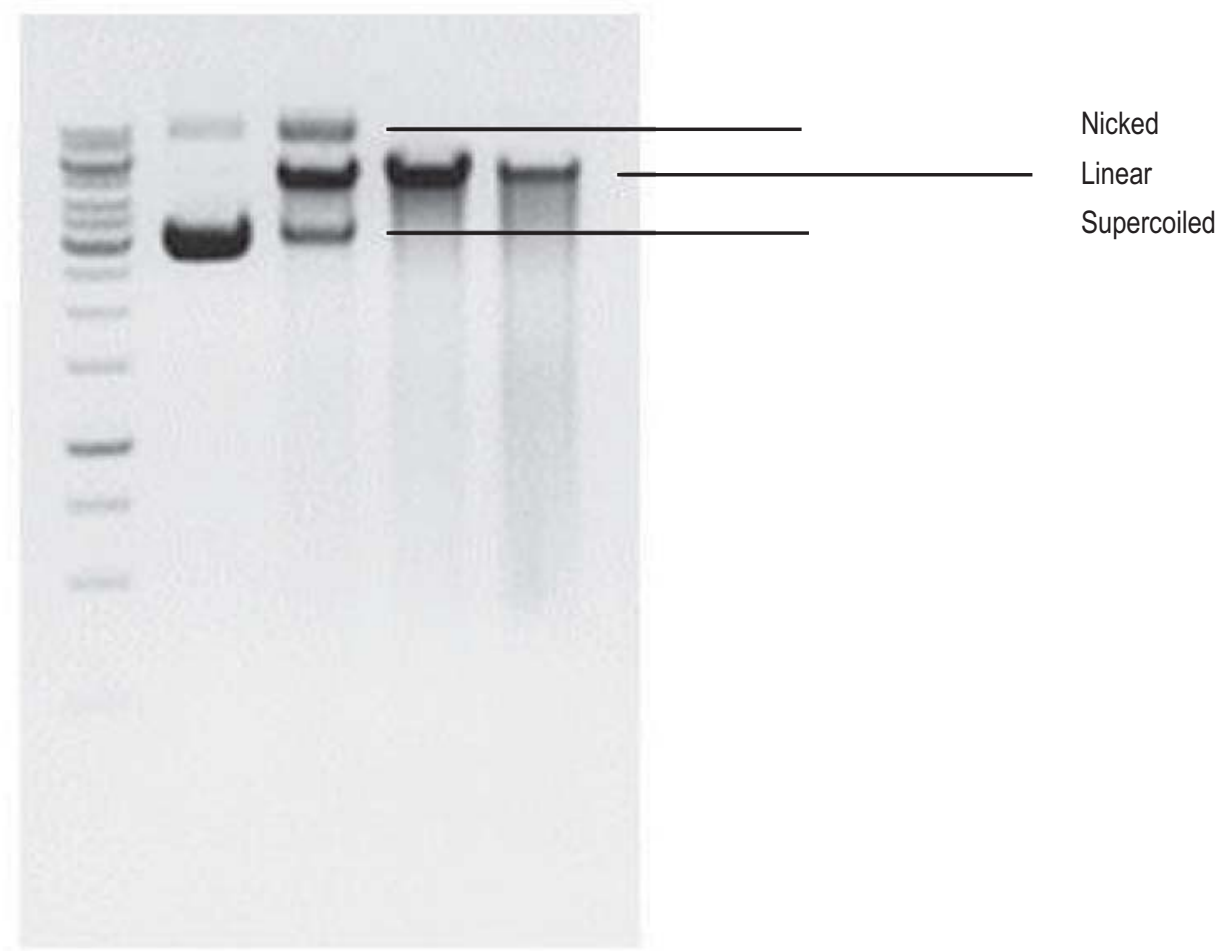

Fig.5 : Restriction profile of phi x 174 circular DNA incubated with NspV like endonuclease.

Lane M - Fermentas DNA ladder $(0.25,0.5,0.75,1,1.5,2,2.5,3,3.5,4,6,8,10 \mathrm{~kb})$ Lane 1-Uncut phi x 174 DNA, lane 2 - phi x 174 DNA cut with NspV like endonuclease
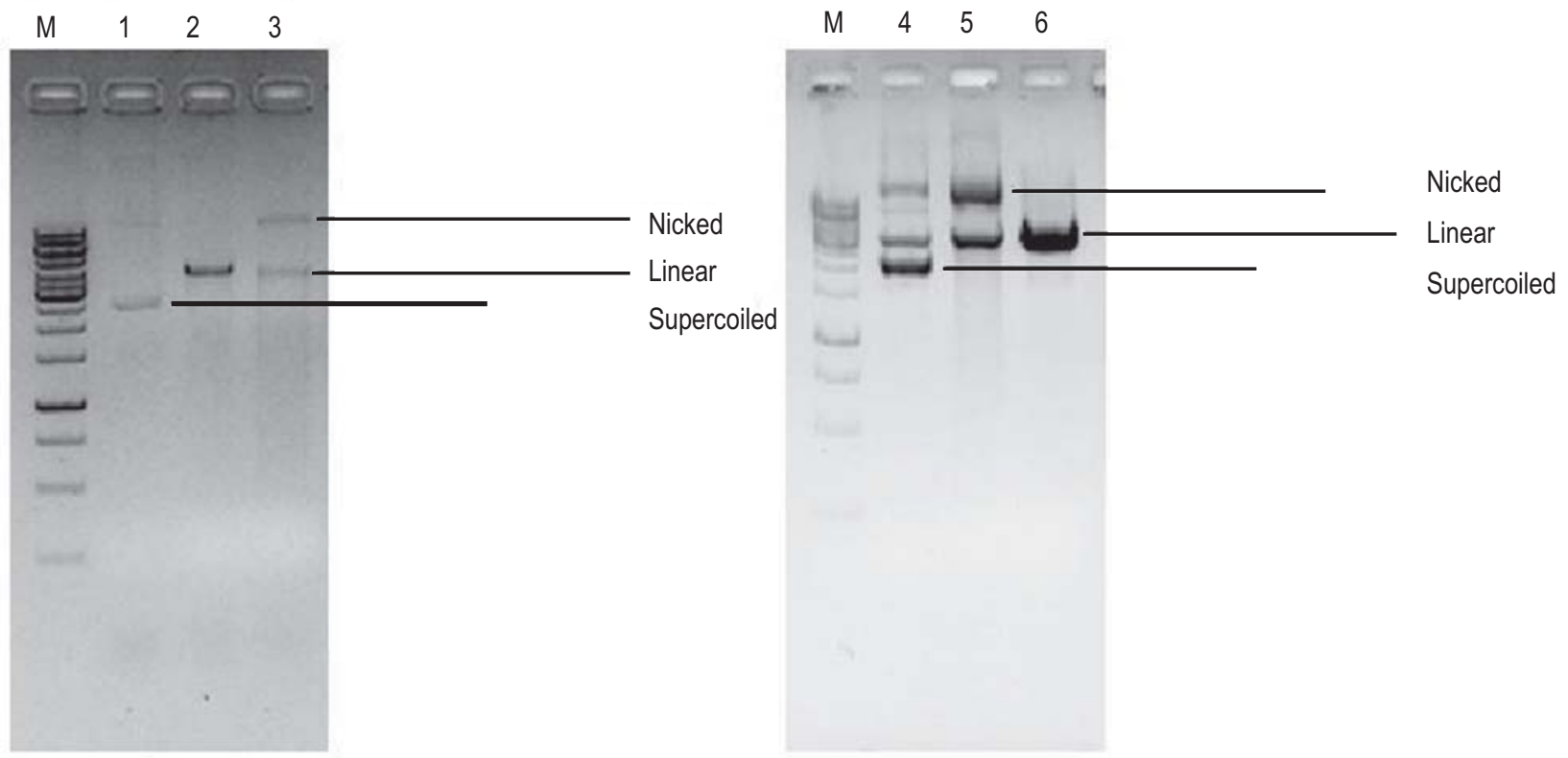

Fig.6 : pACYCDUET and pTZ57R plamid cut with NspV like Restriction Enzyme.

Lane M - Fermentas DNA MW marker $(0.25,0.5,0.75,1,1.5,2,2.5,3,3.5,4,6,8,10 \mathrm{~kb})$ Lane 1-Uncut pACYCDUET-1 plasmid Lane 2-pACYCDUET-1 vector cut with Sac I Lane 3- pACYC-DUET-1 vector cut with NspV like Restriction enzyme Lane 4-Uncut pTZ57R plasmid Lane 5 - pTZ57R plasmid cut with NspV like Restriction Enzyme Lane 6 - pTZ57R cut with EcoRI 
Nicking endonucleases recognize specific sequences and create nicks in DNA (Higgins et al., 2001; Xu et al., 2001). The endonuclease characterized in this study, resulting in a mixture of nicked plasmid (major product observed) and linear plasmid, may be a nicking endonuclease. Double digest of Aeromonas NspV like endonuclease and Xho I or Sac II resulted in a single, linear form of plasmid DNA(Fig. 5) with nicked form dis-appearing completely. Similar nicking endonucleases profiles have been observed in other studies resulting in a mixture of nicked plasmid (major form) and linear plasmid as seen with our enzyme (Lee at al., 2015; Somyoonsap et al., 2013; Higgins et al., 2001). Incubation of various concentrations of Helicobacter pylori HP0268 nuclease, characterized to have nickase activity, with plamsids like $p E T 15 b(+), p E T-21 a(+)$ and pET28a(+) resulted in open circular (nicked) and linear forms of DNA (Lee et al., 2015). Similarly, incubation of Streptomyces DC13 nuclease or nickase, with plasmids pBluescript, pUC18, pET15b, pET26b resulted in accumulation of predominantly Open Circular form and low levels of linear form (Somyoonsap et al., 2013). Another nicking endonuclease, $\mathrm{N}$. BstBI was shown to produce major, open circular form and minor, linear form upon its activity on plasmid substrate (Higgins et al., 2001). The NspV like endonuclease, in this study, on incubation with circular DNA like Phi X 174, pACYC DUET and pTZ57R showed a predominant, open circular form and minor, linear form indicating that it is a nickase.

The conserved domain and amino acid sequence data suggests that it is similar to restriction endonuclease and has been reviewed extensively, nicking enzymes are similar to restriction endonucleases (Zheleznaya et al., 2009; Higgins et al., 2001; Xu et al., 2001). Nickases Nb.BsrD1 and Nb.Btsl have been enginnered from their restriction enzymes, BsrD1 and Btsl (Xu et al., 2007). Along similar lines, a nickase N.Alwl has been engineered from its restriction endonuclease, Alwl (Xu et al., 2001). The comparison of restriction pattern of restriction endonucleases Plel / Mlyl and nickase, N. BstBl shows that they function in similar manner on plasmid substrates (Higgins et al., 2001). Most of the well characterized nicking endonucleases are larger in size $(\sim 38$ or 56 or $70 \mathrm{kDa}$ ) while the NspV like endonuclease described in our study is smaller $(\sim 25 \mathrm{kDa})$. The smaller sized nicking endonucleases could be of interest to the industry with novel applications involving better stability, increased specificity, easier access to site of action and immobilization. Further work needs to be carried out to characterize the cleavage site, the optimal cleavage conditions and potential applications.

\section{Acknowledgements}

The authors would like to thank Syngene Higher Education Program for partially funding this project.

\section{References}

Chan, S.H., B.L. Stoddard and S.Y. Xu: Natural and engineered nicking endonucleases - from cleavage mechanism to engineering of specificity. Nucl. Acids Res., 39, 1-18 (2011)

Davies, G.P., I. Martin and S.S. Sturrock: On the structure and operation of Type I DNA restriction enzymes. J. Mol. Biol., 290, 565-579 (1999).

Drozdetskiy, A., C. Cole, J. Procter and J. E. Geoffrey: JPred4: a protein secondary structure prediction server. Nucl. Acids Res., 43, W389 -W394 (2015).

Higgins, L.S., C. Besnier and H. Kong: The nicking endonuclease N.BstBI is closely related to Type lls restriction endonucleases Mlyl and Plel. Nucl. Acids Res., 29, 2942-2501 (2001)

Janda, J.M. and S.L. Abbott: The Genus Aeromonas: Taxonomy, pathogenicity and infection. Clin. Microbiol. Rev., 23, 35-73 (2010)

Janda, J.M. and S.L. Abbott: Human Pathogens. In: The genus Aeromonas (Eds. : B.Austin, M. Altwegg, P.J. Byosling and S. Jeseph). John Wiley \& Sons Ltd., West Sussex, England, p.151$173(1996)$.

Kobayashi, I. Behavior of restriction-modification systems as selfish mobile elements and their impact on genome evolution. Nucl. Acids Res., 29, 3742-3756 (2001)

Lee, et al.,: Structure-based functional identification of Helicobacter pylori HP0268 as a nuclease with both DNS nicking and RNase activities. Nucl. Acids Res., 43, 5194-5207 (2015)

Luria, S.E. and M.L. Human: A nonhereditary, host-induced variation of bacterial viruses. J. of Bact., 64, 557-569 (1952).

Murray, N. E.: Type I Restriction systems: Sophisticated Molecular machines (a Legacy of Bertani and Weigle). Microbiol. Mol. Biol. Rev., 64, 412-443 (2000)

Pigoud, A. and A. Jeltsch: Recognition and cleavage of DNA by Type II restriction endonucleases. Eur. J. Biochem., 246, 1-22 (1997).

Roberts, et al.,: A nomenclature for restriction enzymes, DNA methyltransferases, homing endonucleases and their genes. Nucl. Acids Res., 31, 1805-1812 (2003).

Somyoonsap, P., V. Kitpreechavanich and S. Pornbanlualap:A sequence specific nicking endonuclease from Streptomyces: Purification, physical and catalytic properties. ISRN Biochemistry., doi:10.1155/2013/287158 (2013).

Xiaoli, D., P. Stothard, I.J. Forsythe and D.S. Wishart: PlasMapper: A web server for drawing and auto-annotating plasmid maps. Nucl. Acids Res.,32, W660-664 (2004).

$\mathrm{Xu}$, Y., K.D. Lunnen and H. Kong: Engineering a nicking endonuclease N.Alwl by domain swapping. Nucl. Acids Res., 23, 12990 - 12995 (2001).

Xu, S. Y., Z. Zhu, P. Zhang, S. H. Chan, J. C. Samuelson, J. Xiao, D. Ingalls and G. G. Wilson: Discovery of natural nicking endonucleases $\mathrm{Nb}$.BsrDl and $\mathrm{Nb}$. Btsl and engineering of top strand nicking variants from BsrDI and Btsl. Nucl. Acids Res., 35, 4608-4618 (2007).

Zheleznaya, L A., G.S. Kachalova, R.I. Artyukh, A.K. Yunusova, T.A. Perevyazova and N.I.Matvienko: Nicking endonucleases. Biochem., (Moscow)., 74, 1457-1466 (2009). 